\title{
L'archi-road movie, ou le routage des sens
}

\author{
Peter Szendy
}

Cada film abre un mundo (o un cinemundo, como dice Jean-Luc Nancy). Por esto, el camino, y el road-movie como género, son más que meros tropos, más que simples metáforas para la película: son los sensorimotor schemata del cine pavimentando su propio camino. El problema de la sincronización por lo tanto se reformula como routing de los sentidos, cada vez en un sentido singular, como su colisión, donde sus trayectorias se cruzan. El artículo analiza el curso filmado por los ojos y oídos de un número de films como Blow Out (Brian De Palma, 1981), Lost Highway (David Lynch, 1997), and Deathproof (Quentin Tarantino, 2007).

PALABRAS CLAVE: road-movie, visual, auditivo, cine.

Each film opens a world (or a cineworld, as Jean-Luc Nancy says). This is why the road, and the road-movie as a genre, are more than mere tropes, more than simple metaphors for the motion picture: they are the sensorimotor schemata of cinema's paving its own pathway. The question of synchronization is therefore reformulated as the routing of the senses, each time in a singular way, and as their collision, where their trajectories cross. The article analyzes the courses taken by the eyes and ears in a number of films, including Blow Out (Brian De Palma, 1981), Lost Highway (David Lynch, 1997), and Deathproof (Quentin Tarantino, 2007).

KEYWORDS: road-movie, visual, auditive, cinema.

Fecha de recepción: 20 de junio de 2013

Fecha de aceptación: 2 de septiembre de 2013 



\section{Peter Szendy}

Universidad Paris X Nanterre

\section{L'archi-road movie, ou le routage des sens}

En 1966, Antonioni filmait dans Blow-Up un photographe de mode qui, développant et agrandissant ses clichés d'un couple amoureux surpris dans un parc, fait apparaître l'indice d'un meurtre. Quinze ans plus tard, en 1981, De Palma réalise avec Blow Out une sorte de remake dans lequel la photographie est remplacée par la phonographie, le protagoniste étant cette fois un bruiteur et preneur de son.

Mais ce qui, au-delà de l'enquête, sera le nœud de l'intrigue, c'est la fabrique même du cinéma en tant que greffe audio-visuelle. ${ }^{1}$ C'est-à-dire aussi, on le verra, en tant que ce deuil de la synchronie qui, appelant dès lors une autre pensée de la coïncidence, devra nous conduire (c'est le mot) vers l'hypothèse d'un routage des sens.

On pourrait en effet dire de Blow Out qu'y est à l'œuvre une déconstruction du concept même d'audio-visuel: à la fois son démontage, la mise en scène de son impossibilité (la coïncidence, la coprésence qu'il implique est irrémédiablement différée et emportée), ainsi que la réinscription endeuillée, pourtant, de son nécessaire fantasme et de ses effets. C'est pourquoi Blow Out, cette histoire d'accident de la route,

${ }^{1}$ Avec son installation vidéo intitulée Up and Out (1998), Christian Marclay, de façon joueuse et sérieuse à la fois, invite à la confrontation de l'original et du remake. En une sorte de greffe cinématographique croisée, $U p$ and Out superpose en effet les images de Blow-Up à la bande-son de Blow Out, avec de saisissants effets de décalage, notamment à la fin, lorsque les dernières images d'Antonioni se déroulent dans un silence de mort, dû à la différence de durée des deux films. 
sera ici un prélude obligé pour interroger plus généralement le cinéma comme frayage de l'expérience où la vue et l'écoute ne cessent de se frôler, se toucher, se heurter, bref, de s'accidenter l'une l'autre. Et, nous $\mathrm{y}$ viendrons, le théâtre de ce qu'il faudra dès lors appeler leurs accrochages (dans tous les sens possibles de ce mot), c'est par excellence le road movie: non pas en tant que genre ou répertoire constitué, mais en tant que mise en scène chaque fois singulière de l'ouverture d'un (ciné) monde. ${ }^{2}$

Les premières images de Blow Out sont un faux prologue. On croit être dans un film d'horreur flirtant avec un érotisme bon marché: dans une résidence pour jeunes filles, l'ombre d'un maniaque rôde et finit par s'approcher de l'une d'elles, nue sous sa douche. Dans cette citation presque littérale de $P$ sycho, ${ }^{3}$ on voit le bras assassin qui écarte le rideau de plastique, la main qui brandit le couteau meurtrier et, enfin, le visage de la victime qui pousse un cri...

Changement brusque de décor: dans un studio de montage, le bruiteur, Jack Terry (John Travolta), discute avec le réalisateur de ces piètres séquences. Le cri de la jeune fille n'est pas convaincant, il faut en dénicher un meilleur. On comprend qu'il s'agissait d'un film dans le film: le cinéma parle ici de lui-même, de l'improbable synchronie de l'image et du son.

C'est alors qu'intervient le générique de début.

Les noms défilent sur un écran dédoublé, en split screen: à droite, Jack qui regarde les informations télévisées sur la campagne du gouverneur McRyan, candidat aux élections; à gauche, gros plans sur ses magnétophones et tables de mixage. De même que les plans du film dans le

${ }^{2}$ Sur le road movie en général, $c f$. notamment Devin Orgeron, Road Movies : From Muybridge and Méliès to Lynch and Kiarostami; et David Laderman, Driving Visions. Exploring the Road Movie.

${ }^{3}$ De la fameuse séquence au cours de laquelle Norman Bates (Anthony Perkins) assassine Marion Crane (Janet Leigh) dans la douche du motel. 
film, les faits que les nouvelles rapportent et montrent seraient-ils faits, c'est-à-dire fabriqués, assemblés, montés?

Fin du générique.

Jack sort de son studio. Il fait nuit. Il est dehors, dans la nature, sur le pont traversant la rivière. Pour se distraire, avec un plaisir et une curiosité visibles, il enregistre les sons les plus variés qu'il capte avec un long microphone directionnel, dont la caméra montre avec insistance la pointe, sorte de tête chercheuse ou de sonde. Chaque fois, le son précède l'image: le vent à travers les feuillages; un couple qui passe (elle remarque Jack sur le pont, se demande si c'est un "voyeur", a Peeping Tom); un crapaud, puis un hibou... Jack a l'air amusé, comme un grand enfant jouant avec un sonar. Mais lorsqu'il entend soudain dans les oreillettes de son casque des crissements de pneus au loin, son expression change. Il dresse l'oreille, il a lui-même quelque chose d'animal dans son être aux aguets. Le potentiomètre de son magnétophone décrit déjà la course d'une voiture que l'on ne voit pas encore. On entend un bruit d'explosion (blow-out), et voilà le véhicule qui apparaît à l'écran, lancé à vive allure sur le pont: il fait une embardée, heurte les barrières, tombe et s'enfonce lentement dans l'eau...

Dès ces dix premières minutes, dès ces séquences d'ouverture, avant même qu'une intrigue ne se noue, Blow Out est donc une saisissante mise en scène du montage audio-visuel comme trait d'union sans cesse retracé et différé. Soit l'image est déjà là et le son ne parvient pas à s'y ajuster (c'est le cas du cri sous la douche). Soit l'oreille (celle de Jack), appareillée de prothèses microphoniques, entend d'avance et de loin ce que l'œil ne perçoit pas encore.

La suite de Blow Out n'est que le vaste déploiement narratif de ce discord entre le visible et l'audible.

En se jetant à l'eau, Jack a réussi à extraire de la voiture Sally (Nancy Allen), mais le conducteur, qui n'était autre que le gouverneur McRyan, meurt. Peu à peu, en réécoutant ses enregistrements in situ, Jack soupçonne un crime plutôt qu'un accident. Il croit entendre un coup de feu sur ses bandes, mais il ne parvient pas à l'identifier, car le bruit en est masqué par l'explosion et le crissement des pneus. Bref, il y a un masque sonore, et c'est seulement en ayant recours à l'archive visuelle de 
l'accident que Jack pourra véritablement déceler ce qu'il soupçonne déjà et entrevoit (entrécoute, devrait-on dire).

En effet, Jack découvre que le gouverneur faisait l'objet d'une machination politique visant à le discréditer. Un photographe, un pauvre type du nom de Karp (Dennis Franz), devait prendre de lui des clichés compromettants avec Sally, qui s'était prêtée au jeu. Aussi Karp a-til photographié en rafale leur passage en voiture sur le pont. Lorsque Jack découvre les photogrammes publiés dans la presse, il décide de les monter en une séquence qu'il sonorise avec ses bandes. Ainsi, la synchronisation révélera précisément, sur l'une des images, la trace de la lueur d'une arme à feu. Il en a désormais la preuve: visant le scandale, la machination, pour des raisons obscures, a tourné à l'assassinat.

L'auteur du meurtre, le sinistre Burke (John Lithgow), cherche désormais à éliminer Sally qui, en tant que survivante, est devenue un témoin dangereux. Mais Jack, quant à lui, veut utiliser Sally pour remonter la piste jusqu'à l'assassin. Dans la longue et mémorable séquence finale, sur fond de feux d'artifice (c'est le jour de la fête de l'Indépendance, à Philadelphie), Sally rencontre Burke. Jack les écoute à distance, grâce à l'appareillage de radiotransmission qu'il a caché sous les vêtements de Sally. Et c'est ainsi que, perdu dans la foule des festivités, il entendra de loin les appels au secours de la jeune fille, puis son terrible cri lorsqu'elle meurt sous les coups de Burke. Les dernières images montrent Jack désespéré de n'avoir pu la sauver: il réécoute de façon obsessionnelle l'enregistrement du cri de sa mort, qu'il finit par utiliser pour sonoriser la scène sous la douche du film dans le film. Un vrai cri, convaincant. A good scream. Enfin.

\section{II}

Blow Out est donc d'abord un film sur le désajointement de l'audible et du visible. Le son est toujours en avance ou en retard, il ne colle jamais à l'image. A l'exception de deux instants ou incidents: celui du coup de feu dans le photomontage sonorisé de l'accident; celui du cri de Sally, greffé in fine sur l'image de la jeune fille dans sa douche. 
En montrant ainsi le travail de synchronisation à l'écran, dans l'histoire, en jouant à brouiller la frontière entre le dedans et le dehors de la diégèse, ce que semble vouloir mettre en scène ce remarquable thriller, c'est que l'audiovisuel, comme on dit, n'est qu'une sorte de dangereux glissement métonymique affectant la bande-son et l'image, l'une par rapport à l'autre. Leur loi, c'est le décalage. C'est un écart qui diffère l'une par et dans l'autre: le visible témoigne du secret de l'audible, et inversement.

Mais Blow Out est aussi, du même coup, un grand film sur l'écoute espionne ou de surveillance, mise en scène sous la forme d'un double mouvement dans l'audition. ${ }^{4} \mathrm{Car}$, dans sa pulsion de captation, l'écoute policière implique toujours l'avance ou le retard au regard du visible: elle s'oriente vers l'indice de ce qui, pour l'œil, n'est pas encore ou déjà plus là. En ce sens, elle est structurellement défectueuse, en défaut (c'est pourquoi, d'ailleurs, elle semble appeler à l'infini son appareillage prothétique).

Entre cette écoute défaillante des mortels espions que nous sommes —nous, êtres finis toujours prêts à dresser l'oreille- et l'écoute à mort qui nous promet une synchronie totalisante, c'est un peu la vieille histoire d'Orphée que Blow Out raconte ainsi à nouveau.

Orphée, en effet, aura tué Eurydice par une sorte de surenchère inscrite dans son oreille mortelle: sa peur de ne pas ouïr tandis qu'il marche et chante le pousse à se tourner vers la vision qui lui est interdite, comme si la pulsion scopique, voire panoptique, promettait cette totalisation que l'oreille défectueuse se voit sans cesse déniée. Et c'est un phonodrame analogue, c'est une ototragédie audiovisuelle à tant d'égards semblable que vit Jack dans Blow Out, entre l'instant où il aura plongé dans l'eau pour sauver Sally de la noyade (l'arrachant au Styx) et celui où il la fait mourir à nouveau en l'écoutant sans pouvoir la voir.

Quant au cri final, c'est le hurlement de la mort dans et comme la synchronie même. C'est le cri de terreur que recèle l'accident de la coïncidence parfaite. C'est l'arrêt de mort dans l'instant fantastique et fantas-

${ }^{4}$ J'ai tenté de développer, dans Sur écoute. Esthétique de l'espionnage, les conséquences générales d'une telle duplicité —ou diaphonie — logée au cœur de l'écoute. J'y propose aussi une analyse de l'Orfeo de Monteverdi qui pourrait être lue en parallèle avec Blow Out. 
matique où se résorbe la disjonction, dans le clin d'œil où plus rien ne diffère. Quand le son colle à l'image, quand il est présent à elle, quand il est là, ci-devant, synchronisé à mort, c'est alors que tout se renverse et se retourne dans l'infini du différé, dans le décalage absolu sans résorption possible, dans la perte et le deuil.

À partir du deuil de la synchronie que nous conte cette fable otoroutière qu'est Blow Out - l'audiovisuel, comme tel, y apparait en effet comme un accident de la route-, je voudrais tenter d'avancer quelques pistes en direction de ce que j'appellerai le routage des sens.

Si je devais choisir un mot, un seul et unique mot d'ordre pour ramasser la trajectoire qui nous attend maintenant, ce serait sans doute celui-ci: Blickbahn. Ce mot allemand est inhabituel, il est insolite, comme le note Jean-Luc Nancy: "terme rare, dont la lettre signifierait "frayage du regard" (Nancy, La Déclosion, 161). Ce signifiant inusuel, on le trouve pourtant ici ou là chez Heidegger: non seulement dans le $\S 42$ des Beiträge auquel Nancy se réfère, mais aussi, par exemple, dans Introduction à la métaphysique (à partir du $\$ 45$, où il apparaît également pourvu d'un préfixe: Vor-blickbahn, pour dire une sorte de préperspective qui s'est déjà ouverte avant que le regard n'y plonge), ${ }^{5}$ ou encore dans L'Origine de l'œuvre d'art, dans Der Satz vom Grund...

C'est donc ce terme étrange que je voudrais prendre pour devise ou guide (Leitwort, pourrait-on dire dans la langue de Heidegger), c'est en suivant son frayage que je nous laisserai conduire, entraîner ou embarquer dans ce qui ressemblera à un étrange road movie.

Road movie, du reste, ce pourrait être une traduction, certes maladroite ou fautive en apparence, de Blickbahn.

${ }^{5}$ Martin Heidegger, Einführung in die Metaphysik (Gesamtausgabe, II, 40), Vittorio Klostermann: Die Blickbahn des Anblicks muss im voraus schon gebahnt sein. Wir nennen sie die Vor-blickbahn, die „Perspektive“. Gilbert Kahn traduit ainsi (Introduction à la métaphysique, Gallimard): "Le champ d'inspection de ce que nous voyons doit être déjà ouvert d'avance. Nous le nommons le champ préalable d'inspection, la 'perspective", 
Mettons des images sur ce mot de Blickbahn.

Imaginons: que serait un film dont le scénario se réduirait à ce seul et insolite lexème?

On y verrait et entendrait peut-être ce qu'on entend et voit au générique de Lost Highway de David Lynch (1997): un bruit de vent et de moteur qui tourne, l'éclairage des phares, le défilement à folle allure d'une ligne jaune discontinue marquant les deux voies d'une route asphaltée qui s'enfonce dans la nuit. Pendant que les noms des acteurs et le titre s'affichent à l'écran telles des formes qui s'écrasent sur un parebrise, en caractères jaunes comme la signalétique routière, on entend la voix de David Bowie dans I'm deranged (sur l'album Outside en 1995), cette voix qui flotte de façon étrangement inquiétante sur fond de rythmique débridée et l'on devine aussi en les attrapant au vol des bribes de paroles chantées, how secrets travel, comment les secrets voyagent, cruise me babe, drague-moi, roule-moi ou conduis-moi... L'écran vire peu à peu au noir total: fin du générique, le film peut commencer. ${ }^{6}$

Que regarde-t-on, avant même que Lost Highway n'entreprenne de raconter ceci ou de montrer cela, que voit-on dans la dromoscopie déchaînée de ce générique presque abstrait? Chaque fois que je me repasse ces hypnotiques images sans images, je me dis: c'est comme si, de tous les road movies de l'histoire du cinéma - depuis Le Salaire de la peur de Clouzot (1953) jusqu'à The Road de John Hillcoat (2009), en passant par le mythique Easy Rider de Dennis Hopper (1969) ou Duel de Spielberg (1971)—, c'est comme si de tous ces films routiers, donc, on retirait les personnages et leurs vécus, les paysages, les péripéties, les arrêts et les départs, les stations et les surprises ou les revirements qu'elles réservent, pour ne garder que... Que quoi? Peut-être la simple forme d'un cadre en déplacement qui soit à même de produire la figure

${ }^{6}$ Cf. David Lynch et Barry Gifford, Lost Highway. Scénario, Cahiers du cinéma, núm. 9: "Scène 1. Ext[érieur]. Route à deux voies - nuit. Nous suivons un point de vue très net, illuminé par des phares. Nous flottons au-dessus d'une vieille route à deux voies qui traverse un paysage désolé de désert. Ce point de vue effrayant et lumineux continuera tout au long du générique. À la fin du générique, les phares semblent baisser d'intensité et très vite nous nous retrouvons dans le noir". 
purement cinématique de la route. L'archi-scénario du Blickbahn, en somme.

Dans L'Évidence du film, Nancy décrit ainsi le dispositif du cinéma (L'Évidence du film, 15):

Le regardeur est fixé à une place dans l'obscurité d'une salle dont on ne peut pas dire que l'image filmique s'y trouve (comme une autre image pourrait le faire), puisqu'elle constitue en vérité tout un côté de cette salle. C'est ainsi la salle elle-même qui devient lieu ou dispositif de regard, boîte à regarder — ou plutôt: boîte qui est ou qui fait regard comme on dit, en français, "un regard" pour désigner une ouverture destinée à permettre une observation ou une inspection (dans une canalisation, dans une machine). Ici le regard est une entrée dans un espace, il est une pénétration avant d'être une considération ou une contemplation.

Le regard filmique entre, pénètre, ouvre, fait effraction à la façon de l'opération chirurgicale qui, pour Benjamin déjà, constituait le paradigme du mouvement de la caméra. ${ }^{7}$ Et c'est sans doute en vertu de ce caractère endoscopique que le cinéma, comme l'écrit encore Nancy,

${ }^{7} C f$. Walter Benjamin, "L'CEuvre d'art à l'époque de sa reproductibilité technique" (dernière version de 1939), traduction française de Maurice de Gandillac revue par Rainer Rochlitz, dans Euvres, III, Gallimard, coll. "Folio": "Entre le peintre et le cameraman nous retrouvons le même rapport qu'entre le mage et le chirurgien. Le peintre observe, en peignant, une distance naturelle entre la réalité donnée et lui-même ; le cameraman pénètre en profondeur dans la trame même du donné." Benjamin ajoute en note : "Les hardiesses du cameraman sont effectivement comparables à celles du chirurgien" (lequel, pouvait-on lire quelques lignes plus haut, "renonce à s'installer en face du malade [...] ; c'est plutôt opérativement qu'il pénètre en lui"). Dans le cinéma récent, c'est sans doute Panic Room de David Fincher (2002) qui, grâce au renfort des effets numériques, démontre avec le plus de virtuosité cette puissance chirurgicale de la caméra. Lorsque Burnham (Forest Whitaker) et Junior (Jared Leto) s'apprêtent à faire effraction dans la maison qu'occupent Meg (Jodie Foster) et sa fille Sarah (Kristen Stewart), la caméra entre d'abord dans le trou de la serrure, que les cambrioleurs ne parviennent pas à ouvrir. La caméra ressort et les montre en train de scruter la pièce du dehors, par la fenêtre, avant qu'ils ne se décident à chercher un autre accès. La caméra fait alors demi-tour, elle traverse la pièce en rasant les meubles et les objets, passant dans l'anse de la cafetière électrique... 
est "bien plus que l'invention d'un art surnuméraire": il est "la poussée d'un schème de l'expérience" (21).

Cette poussée, c'est celle d'un mouvement qui ne traverse pas des lieux déjà établis et distribués, car il les constitue plutôt: "Le mouvement me porte ailleurs mais 1'“ailleurs" n'est pas préalablement donné: c'est ma venue qui en fera le "là-bas" où je serai venu d'“ici" (p29). Une telle cinématique de la vue, qui dispose et structure ce qu'elle traverse au fil de sa traversée, c'est le cinéma en tant que frayage du regard, Blickbahn. C'est le tracé ouvrant d'une préperspective qui rend l'expérience cinématographique possible, qui fait qu'il y a ce "cinémonde" dont parle Nancy:

[...] le cinémonde est un monde, le nôtre, dont l'expérience est schématisée - au sens kantien, c'est-à-dire rendue possible dans sa configuration - par le cinéma. Cela ne veut pas dire que notre monde ne répondrait qu'à ce schématisme, mais il le compte au nombre de ses conditions de possibilité. Lorsque nous regardons un paysage d'un train, d'un avion ou d'une voiture, ou bien lorsque nous fixons soudain un objet, un détail sur un visage ou bien un insecte, dans un certain mouvement d'approche du regard, lorsque nous découvrons la perspective d'une rue, lorsque nous apprécions une situation remarquable, étrange, surprenante ou déconcertante, mais aussi en buvant un café ou en descendant l'escalier, autant d'occasions sont tendues de penser ou de dire "c'est du cinéma".

Or, la voiture qui est mentionnée ici n'est pas seulement un exemple parmi d'autres, l'une quelconque parmi toutes ces situations où se produit une cinématisation de la vie. La voiture, ou plutôt le road movie, c'est la réinscription de la condition de possibilité de l'expérience cinématographique dans le cinémonde même: c'est le pli ou la re-marque — quasi-transcendantale, pourrait-on dire avec Derrida — du cinéma. ${ }^{9}$

8 Jean-Luc Nancy, “Cinéfile et cinémonde", Trafic, n 50, P. O. L. Dans ces mêmes pages, Nancy va jusqu'à affirmer que le cinéma n'est rien de moins qu"'un "existential" au sens de Heidegger : une condition de possibilité de l'exister"

${ }^{9} \mathrm{Cf}$. parmi tant d'autres textes où Derrida parle du quasi-transcendantal, ce passage de Résistances - de la psychanalyse: "la loi quasi transcendantale de la sérialité qui trouverait à s'illustrer [...] chaque fois que la condition transcendantale d'une série fait aussi partie, paradoxalement, de la série". C'est peut-être également ainsi qu'il faut lire ces lignes de Nancy lorsqu'il récuse pour le cinéma la pertinence du paradigme de la 
"La voiture qui roule", écrit Nancy dans L'Évidence du film, "est donc aussi deux fois une vérité cinématique: une fois en tant que boîte à regard, et une fois en tant que mouvement incessant" (29). Aussi le road movie n'est-il pas un genre cinématographique parmi d'autres, mais le genre pour lequel il y va du cinéma comme tel. C'est au fond ce que Nancy, sans le formuler explicitement ainsi, ne cesse de dire à propos des films de Kiarostami: il décrit "le déplacement d'une voiture, de l'objectif en elle et hors d'elle: un film dont le sujet défile comme sa pellicule" (11); il souligne toutes les fois où le réalisateur montre la "fenêtre d'une voiture", un "plan dans un rétroviseur" (17), il note ces "fenêtres de voiture [...] qui ouvrent (amorcent) le film" (15); il parle de "l'objectif que devient une voiture" (31), de "l'image inlassable de la voiture, de ses fenêtres, pare-brise et rétroviseur comme autant de capteurs de vues" (39) ou "capteurs d'images" (51). Bref, comme il l'écrit à propos de Et la vie continue (1992), "l'auto [...] est l'objet central et le sujet du film, son personnage et sa boîte obscure, sa référence et son moteur" (67).

La voiture apparaît dès lors comme l'embrayeur du film - de chaque film où elle apparaît - sur le schème routier, sur le routage qui règle l'expérience filmique comme telle (53, je souligne):

"La conduite de la voiture a une grande importance: tourner le volant, sur ces routes en zigzag, changer de vitesse, serrer le frein. Il se produit un embrayage du film sur cette mécanique cinétique qui requiert l'attention du conducteur".

Voilà pourquoi le Blickbahn, ce tracé ou traçage de la vue, se laisse sans cesse traduire et retraduire dans ce qu'il faudrait appeler l'archiroad movie, architrace du regard filmique ouvrant la préperspective du cinémonde (67):

caverne platonicienne (L'Évidence du film, 45-47, je souligne): “Jusqu'à nous la paroi aux images était consistante et elle témoignait pour un dehors ou pour un tréfonds du monde [...]. Avec le cinéma, la paroi devient une ouverture pratiquée dans le monde sur ce monde même. C'est pourquoi la comparaison, qui fut faite à plusieurs reprises, du cinéma avec la caverne de Platon n'est pas pertinente : le fond de la caverne témoigne précisément pour un dehors du monde, mais en négatif, et installe par là même la déconsidération des images que l'on sait, ou l'exigence de considérer des images plus hautes et plus pures, nommées "idées". Le cinéma opère à l'inverse : il ne reflète pas un dehors, il ouvre le dedans sur lui-même". 
C'est un registre du frayage permanent: sans cesse, il faut trouver, ouvrir la route [...]. L'auto promène l'écran, ou l'objectif, l'écran-objectif de son pare-brise toujours plus loin, et cet écran n'est justement pas un écran - ni un obstacle, ni une paroi de projection-, mais c'est un écrit, c'est une trace sinueuse, escarpée, poussiéreuse.

Au bout du compte, ce que Nancy donne ainsi à penser lorsqu'il analyse "le regard en tant que transport en avant", c'est, ni plus ni moins, l'ipséité filmique comme rapport à soi se différant dans et par le frayage imageant: “(dé)monstration qu'il n’y aura jamais eu de soi installé en position de spectateur, car un sujet n'est jamais que la pointe aiguë, ténue, d'une avancée que se précède indéfiniment" (67).

Voici le film que donc je suis.

La dromoscopie du générique de Lost Highway est littéralement préfigurée, chez Lynch, dans les images de Blue Velvet (1986), lors de la course éperdue dans laquelle Jeffrey Beaumont (Kyle MacLachlan) est entraîné sous la conduite de Frank Booth (Dennis Hopper, qui semble incarner ici la mémoire de Easy Rider). Tandis que Mulholland Drive (2000) changera de rythme, passera de l'extrême vélocité à une lenteur presque aussi vertigineuse pour s'ouvrir sur la vision d'une automobile qui glisse au ralenti entre les bandes de la route qui serpente dans les hauteurs de Los Angeles, avant d'être violemment percutée par un bolide lancé à toute allure. Le cinéma lynchien oscille ainsi entre les extrêmes de l'équipée sauvage (Wild at Heart, 1990) et de la lente traversée (en 1999, A Straight Story met en scène l'inoubliable odyssée d'une tondeuse à gazon à travers les États-Unis). Or, comme le suggère Lynch lui-même, cette imagerie de la voirie et de la locomotion, ces figures de la route, c'est tout simplement le film comme tel: "Une route", déclaret-il (Entretiens avec Chris Rodley, 204), c'est certes "une avancée vers l'inconnu", mais "c'est aussi la définition du cinéma - les lumières s'éteignent, le rideau s'ouvre et on est parti, sans savoir où on va..." 
Une fois qu'on y prête attention, d'innombrables variations sur ce schème cinéroutier ne cessent de se présenter dans l'histoire du cinéma.

Dans Christine (1983), John Carpenter ne montre pas seulement la ligne jaune discontinue de la route nocturne sur laquelle fuit Buddy (William Ostrander) pour échapper à la meurtrière Plymouth d'Arnie (Keith Gordon). À chaque fois que la prise de vue se retourne vers Christine elle-même, à chaque fois que nous regardons face à face et pour ainsi dire les yeux dans les yeux l'automobile hantée, c'est aussi comme si le film tentait de réaliser le "contrechamp absolu"10 ce qu'on voit, c'est un habitacle noir qui disparait derrière des phares éblouissants; ce qui nous regarde, c'est une "boîte à regard", comme dit Nancy, entourée de projecteurs aveuglants.

Dans Deathproof (2007), Quentin Tarantino fait dire à l'un des personnages, Stuntman Mike (Kurt Russell), ces mots qui sont comme la formule algébrique du cinéma en tant qu'architrace optoroutière: “C'est une voiture-cinéma" (this is a movie car), déclare le cascadeur psychopathe en montrant son véhicule à la jeune Pam (Rose McGowan) qui ne se doute encore de rien; "parfois, quand on tourne un accident, le réalisateur veut mettre la caméra dans la voiture" (sometimes when you're shooting a crash, the director wants to put the camera in the car). La jeune fille prend donc place au sein même de la "boîte à regard" en plexiglas, contre les parois de laquelle elle finira écrasée. Ici comme dans Crash de David Cronenberg (1996), la pulsion de mort va - ou roule - de pair avec la pulsion sexuelle, l'une embrayant sur le drive de l'autre. Mais ce que Deathproof met aussi et surtout en parallèle, c'est le déroulement de la pellicule et le défilement de la route: le film, rayé par endroits (Tarantino joue avec les codes de la mauvaise projection dans les salles dites grindhouse), est traversé à la verticale par une ligne discontinue et tressautante qui devient l'exact pendant visuel des tracés jaunes sur le bitume.

${ }^{10}$ Michel Chion, dans La Voix au cinéma, parle ainsi du "mirage du contrechamp absolu : que les personnages du film pourraient nous voir comme nous les voyons...". Autrement dit: que la caméra, adoptant le point de vue subjectif d'un personnage, se retourne en quelque sorte vers elle-même et, se traversant, vers nous qui regardons le film. 
Deathproof est un road movie éminemment cinéphile, tramé d'allusions explicites ou fugaces à tant d'autres films, ouvrant d'innombrables bifurcations et carrefours dans l'histoire du genre. Ainsi, pendant que Julia (Sydney Tamiia Poitier) envoie un SMs amoureux, on entend la musique de Blow Out. Ou encore, à la fin de la seconde partie, on reconnaît, dans la Dodge Challenger blanche de 1970 dénichée par les trois filles, la voiture même de Kowalski dans Vanishing Point de Richard Sarafian (1971). Et l'on se prend alors à surimprimer aux images routières de Deathproof les inoubliables séquences de ce road movie d'anthologie (que les personnages de Tarantino ne cessent d'ailleurs de citer: Stuntman Mike parle des bons vieux Vanishing Point days, l'une des trois filles s'énerve de voir la cascadeuse insister à vouloir conduire some fucking Vanishing Point car). Vanishing Point, c'est en effet un défilé, un catalogue de routes, de pistes, de croisements, de panneaux de signalisation. Mille motifs routiers et autant de séquences parmi lesquelles je retiens tout particulièrement celle où Kowalski, s'approchant de la frontière du Nevada en étant poursuivi par deux voitures de police toutes sirènes hurlantes, évite de justesse deux camions de la voirie en train de tracer des bandes signalétiques blanches sur le bitume: les véhicules traceurs, du coup, dévoient leur ligne en allant s'arrêter sur l'accotement - le frayage est dévié.

C'est ainsi toute l'histoire du cinéma que l'on se prend à vouloir revoir depuis le schème du Blickbahn. ${ }^{11}$ Avec la première séquence d'Ossessione de Visconti (1943), le néoréalisme lui aussi fraye sa voie en montrant une route filmée depuis l'habitacle d'un camion. Plus tard, dans le genre post-apocalyptique et sur fond d'images surimprimées en noir et blanc, on entend la voix off du début de Mad Max 2 (1981) énoncer solennellement: "sur les routes c'était un cauchemar en ligne blanche, seuls survivraient ceux qui étaient assez mobiles" (on the roads it was a white-line nightmare, only those mobile enough [...] would

${ }^{11}$ S'il est vrai que, en 1896, la locomotive des frères Lumière a fondé le cinéma en fonçant ou fondant sur les spectateurs ahuris de L'Arrivée d'un train à la Ciotat, alors on peut penser que le film a été hanté depuis ses débuts par les rails, les bandes, les pistes en tout genre. $C f$. les belles analyses que Raymond Bellour consacre à ce qu'il faudrait appeler des trains d'images dans Le Corps du cinéma. Hypnoses, émotions, animalités, P. O. L., 44-45 et passim. 
survive). Avec le mouvement optoroutier, il y va en effet de la (sur)vie du film en général: de sa condition de possibilité — qu'il donne à voir, qui le donne à voir.

\section{VII}

Depuis qu'il est aussi devenu sonore, le cinéma apparaît souvent comme une sorte de remake de ce vécu que Michel Chion a joliment raconté dans Le Promeneur écoutant (1993). Dans le second chapitre, intitulé On the Road, le "chroniqueur d'écoutes" se décrit ainsi au volant de sa voiture, traversant les États-Unis. Il met de la musique, elle l'accompagne tandis que défilent devant ses yeux les paysages qu'il parcourt. Et il parle alors "de "l'effet musique de film" bien connu des autoradiophiles", lorsque, par exemple, "nous projetons tout en roulant la musique de Bach sur les panoramas sauvages de la côte..." (43). Cet effet bande-son déteint sur les sites qui se succèdent, il les assemble comme en un film — sans pellicule ni caméra - qui se déroule tandis qu'on roule. ${ }^{12}$

Qu'en est-il donc, dans ce que j'appelle ici l'archi-road movie, du montage audiovisuel, du mouvement conjoint du déplacement autoroutier et de la phonographie amplifiée? Doit-on penser, à côté du Blickbahn, ce qu'il faudrait nommer Hörbahn, un frayage sonore parallèle? Mais s'agit-il de parallélisme, justement, sur ces pistes ou ces voies qui nous emportent déjà dans le cinémonde?

Reprenons la route et prêtons l'oreille.

${ }^{12}$ Michel Chion, Le Promeneur écoutant, 46: “[...] c'est, comme chacun l'a vécu, une expérience euphorisante que de rouler dans une voiture avec du beau son. Un genre cinématographique, le road-movie, est même né de cette sensation [je souligne], illustré par exemple par certains des premiers films de Wenders, ou par de nombreux films américains. C'est justement la loi du genre que la bande musicale - en général une compilation d'airs de rock, de rhythm and blues ou de country — nous y soit donnée dans les mêmes conditions acoustiques que celles dont bénéficient les personnages, c'est-à-dire avec un son proche, tonique et défini, comme si les haut-parleurs de la salle de cinéma étaient branchés directement sur l'ampli du véhicule. Cela induit un nouveau mode de participation, où le spectateur voit défiler, glisser le film devant lui, tout comme les personnages voient filer le paysage qu'ils traversent". 
Dans Vanishing Point, c'est un DJ aveugle, Super Soul (Cleavon Little), qui accompagne avec ses mots et convoie en musique la folle course de Kowalski à travers les Etats-Unis. C'est lui qui, en piratant la fréquence de la police, semble presque pouvoir router le chauffard par l'émission quotidienne qu'il diffuse sur les ondes, selon un dispositif de radiopilotage à distance qui rappelle de loin celui mis en scène par Fritz Lang à la fin du Testament du Docteur Mabuse (1933). Car là aussi on assiste à une course effrénée de voitures: celle de Baum (Oskar Beregi), qui fuit en fonçant droit devant parmi les arbres bordant la route, comme téléguidé dans sa conduite par la voix et la silhouette spectrales de Mabuse (Rudolf Klein-Rogge); et celle du commissaire Lohmann (Otto Wernicke), qui poursuit le psychiatre fou avec l'aide de Kent (Gustav Diessl) comme chauffeur. ${ }^{13}$

Dans ces séquences comme dans le générique de Lost Highway, le tracé des deux voies sur le macadam (Two-Lane Blacktop, c'est aussi le titre d'un beau road movie de Monte Hellman en 1971), le marquage bipartite pourrait évoquer le dispositif audiovisuel comme tel. En effet, la double bande ou piste (track), c'est une figure possible pour le support du cinéma sonore: un ruban de pellicule sur lequel, à côté des photogrammes, court la bande-son (sound-track), un peu comme une bande d'arrêt d'urgence, comme un accotement qui borderait la conduite des images, qui ourlerait le mouvement général par lequel le regard s'enfonce dans l'inconnu. ${ }^{14}$

${ }^{13}$ Il est surprenant que Hitchcock, dans son importante et passionnante interview sur la course-poursuite comme paradigme du cinéma, ne dise rien du rôle de la bande-son. ("Core of the Movie - The Chase", qui a d'abord paru dans le New York Times Magazine du 29 octobre 1950, est repris dans Hitchcock on Hitchcock. Selected Writings and Interviews, $125 \mathrm{sq}$.)

${ }^{14}$ Il faudrait bien entendu prendre en compte ici l'histoire technologique du cinéma, avec les différents procédés de synchronisation entre sons et images qui l'ont jalonnée, et notamment l'adoption de la piste optique courant le long de la pellicule (le procédé dit sound-on-film, développé par Tri-Ergon et Tobis-Klangfilm au début des années trente). Mais, de même que la page reste une unité, voire un concept déterminant sur les écrans déroulants de l'ordinateur, de même la figure de la piste ou bande continue-t-elle de configurer les représentations du sonore dans le contexte d'un film. Exactement comme le mot film lui-même, du reste, lorsqu'il persiste, à l'ère du numérique, à désigner une œuvre audiovisuelle par une métonymie issue d'une technologie antérieure (film, on le sait, veut dire "pellicule" en anglais). 
Est-ce là l'archifilm d'archive de l'audiovision? Serait-ce là la remarque quasi-transcendantale, à l'écran et en split-screen, qui donnerait à écouter-voir les deux frayages parallèles du cinémonde — d'un côté le voir - de l'autre l'écoute — séparés par le tracé clignotant de la ligne jaune - intermittente - qui divise - tout en les assemblant — les deux pistes-ou bandes?

\section{VIII}

Le parallélisme est trompeur. Il est presque aussi naïf que la tranquille et jolie personnification de la piste sonore que proposait telle séquence du premier Fantasia de Walt Disney. Car le schème audiovisuel du cinéma ne s'obtient pas par l'ajout côte à côte de deux voies, défilant l'une contre l'autre dans un frayage synchrone. Pour rester dans le registre du dessin animé, il faudrait peut-être plutôt penser l'expérience de l'écouter-voir cinématographique, avec Tex Avery, comme une sortie, comme une embardée qui fait quitter l'optoroute des photogrammes vers la piste sonore qui la borde.

Pauvre loup! Dans un épisode de 1943 intitulé Dumb-Hounded, il fait tout pour échapper à Droopy (dont c'est d'ailleurs la première apparition à l'écran): il devient une sorte de Kowalski animé, il sillonne le monde entier à toute vitesse, mais il est chaque fois rattrapé par le chien policier. Lorsqu'il s'enfuit de son igloo du pôle Nord, traverse comme une flèche les Etats-Unis et se retrouve à courir désespérément dans une rue de New York, il n'a plus qu'une ultime solution pour tenter de semer le limier qui le traque: sortir du film. Il quitte donc l'image et, tandis qu'il glisse et patine dans le hors-cadre qui constitue le domaine de la bande-son, on entend comme des crissements de pneus.

Dans le Testament du Docteur Mabuse aussi, lorsque les deux voitures, lancées l'une après l'autre dans une course-poursuite effrénée, traversent une voie ferrée à l'instant précis où s'abaisse la barrière du passage à niveau, on aperçoit un panneau qui indique: Achtung! Bahnübergang (littéralement: "attention! franchissement de piste"). Comme si l'articulation du Blickbahn et du Hörbahn devait être placée sous le signe de l'accident de la route, du heurt ou du choc qui menace de se 
produire: tandis que le fantôme de Mabuse en surimpression pointe du doigt la route que Baum doit suivre au volant, la caméra montre avec insistance l'accotement, suggérant l'écart imminent qui fera sortir le véhicule de sa bande.

Sur toutes ces pistes et ces routes, entre les bretelles et les échangeurs, il y a donc la hantise ou le fantasme de l'accrochage. Qui serait le point-limite, le point d'accroche proprement filmique où chaque zone du sentir toucherait, in extremis, à son bord. Car sentir, comme l'écrit Nancy,

c'est toujours sentir à la fois qu'il y a de l'autre (ce que l'on sent) et qu'il y a d'autres zones du sentir, ignorées par celle qui sent en ce moment, ou bien auxquelles celle-ci touche de tous côtés, mais seulement par la limite où elle cesse d'être la zone qu'elle est. Chaque sentir touche au reste du sentir comme à ce qu'il ne peut pas sentir. La vue ne voit pas le son, ni ne l'entend, bien que ce soit en elle-même aussi, ou à même elle-même, qu'elle touche à ce non-voir et qu'elle est touchée par lui...15

15 "Pourquoi y a-t-il plusieurs arts, et non pas un seul ? (Entretien sur la pluralité des mondes)", dans Les Muses. -Il faudrait ici rappeler et lire de près le seul passage, à ma connaissance, où Heidegger parle non pas $d u$ film en général (comme il le fait notamment au tout début de la conférence de 1950 sur La Chose), mais d'un film en particulier, à savoir Rashomon d'Akira Kurosawa (1950). En effet, dans "D'un entretien de la parole. Entre un Japonais et un qui demande" (Acheminement vers la parole, traduction française de François Fédier, Gallimard, coll. "Tel", 1988), il est à la fois question du cinéma comme faire- ou laisser-être-monde et d'un toucher tel que seul le cinéma semble pouvoir le faire advenir. Dans cet étrange dialogue, les deux voix (parmi lesquelles on aimerait sans cesse — c'est irrésistible — pouvoir identifier celle de Heidegger "lui-même") se mettent d'abord à débattre du "réalisme" supposé de Rashomon (102) : “J. — [...] Parlant du réalisme du film (mit dem Hinweis auf das Realistische des Films), je voulais au fond dire [...] que le monde japonais en général (die japanische Welt überhaupt) était capturé dans l'objectivation de la photographie (in das Gegenständliche der Photographie eingefangen), qu'il était proprement forcé à prendre la pose devant elle, bref qu'il était réduit à figurer comme disponible (für diese eigens gestellt ist). D. - Si je vous ai bien prêté attention, vous aimeriez dire que le monde d'ExtrêmeOrient et le produit technique-esthétique de l'industrie cinématographique sont mutuellement incompatibles (die ostasiatische Welt und das technisch-ästetische Produkt der Filmindustrie miteinander unvereinbar sind). J. - C'est exactement cela." Ces propos du Japonais semblent donc faire écho à ceux de Heidegger sur la technique comme productrice d'un non-monde (cet Unwelt dont il est question, par exemple, dans Überwindung der Metaphysik). Un peu plus haut dans le dialogue, on peut lire en revanche cet autre échange, qui semble faire signe au contraire vers la capacité qu'a le cinéma de 
Traduisons ces lignes dans le lexique du schème opto- et otoroutier: non seulement le Blickbahn ouvre la préperspective de ce qui peut dès lors venir à l'image mais il ne peut frayer ainsi la voie de la vue que s'il ne cesse aussi, en même temps, de frôler ce qui se donne à entendre, voire de s'y heurter. Dans un tout autre contexte, Derrida soulignait "qu'il n'y a pas de frayage pur sans différence" (Derrida, "Freud et la scène de l'écriture", 299) — c'est-à-dire, pour ce qui nous importe ici, sans différence des sens. Laquelle, toutefois, ne précède pas le tracé de la route, car il n'y a pas la vue ni l'écoute, dans leur généralité stable, avant qu'elles ne se (re)distribuent, l'une contre l'autre, tout contre, au rythme des cahots et des secousses qui accompagnent la percée du cinémonde.

Nancy fait d'abord l'hypothèse, dans Les Muses, que "la ou les distributions des sens seraient elles-mêmes les produits de l'art" (26). Et il n'hésite pas à affirmer ensuite:

"Chaque œuvre est à sa façon une synesthésie, et l'ouverture d'un monde. Mais c'est en tant que "le monde" comme tel, en son être-monde [...], est pluralité de mondes." (58-59, je souligne). Chaque fois unique, donc, le routage des sens dans les cinémondes.

Et c'est pourquoi il semble difficile de se précipiter à suivre les hérauts d'un "tournant auditif" ou "acoustique" (auditory turn, acoustic turn, aural turn), dans les humanités, dans la théorie, dans la pensée. Certes, il y a eu, indéniablement, un intérêt renouvelé pour le son et

redistribuer le sentir - exemplairement : le toucher — comme s'il provenait d'un autre monde (101) : “D. - J'ai cru y éprouver [dans Rashomon] ce qu'il y a de fascinant (Bezaubernde) dans le monde japonais, ce qui vous emporte en plein secret (in das Geheimnisvolle entführt). Aussi je ne comprends pas pourquoi vous donnez précisément ce film comme exemple de l'européanisation qui consume tout (der alles verzehrenden Europäisierung). J. - Nous autres, Japonais, nous trouvons bien trop réaliste la représentation (Darstellung), par exemple dans les scènes de duel. D. - Pourtant, n'y a-t-il pas aussi des gestes pleins de retenue (verhaltene Gebärden)? J. - Ce genre de choses inapparentes coule à flots à travers le film et de façon à peine perceptible pour le regard européen (Unscheinbares dieser Art fließt in Fülle und kaum merklich für das europäische Betrachten durch diesen Film). Je pense à une main calmement posée (eine aufruhende Hand), en laquelle se rassemble un toucher (in der sich ein Berühren versammelt) qui demeure infiniment loin de tout palper (Betasten), et qui ne peut même plus être appelé un geste (Gebärde), au sens où je crois comprendre ce mot dans l'usage qu'en fait votre langue." 
l'écoute, auquel ou duquel participaient telles pages de Nancy, de moimême, d'autres. ${ }^{16}$ Mais les virages qui dévient, qui dévoient le visible vers l'audible (ou inversement), ces virages ne sauraient être pris ou saisis d'en haut, à vol d'oiseau: si volte-face il y a, elle n'est pas à chercher ailleurs que dans le quasi-transcendantal où se plie le schème opto- et otoroutier sur le tournant qui se produit singulièrement ici et maintenant.

C'est ainsi qu'il faut comprendre Nancy, je crois, lorsqu'il parle de "transimmanence" ("la transcendance d'une immanence qui ne sort pas d'elle-même en transcendant") et lorsqu'il souscrit au mot d'ordre d'Adorno selon lequel "l'esthétique suppose absolument l'immersion dans l'œuvre particulière." (Les Muses, 63).

Chaque fois unique, disais-je, le routage des sens.

\section{RÉFÉRENCES}

Bellour, Raymond, dans Le Corps du cinéma. Hypnoses, émotions, animalités, P. O. L., 2009, 44-45 et passim.

Benjamin, Walter, "L'Euvre d'art à l'époque de sa reproductibilité technique" (dernière version de 1939), traduction française de Maurice de Gandillac revue par Rainer Rochlitz, dans Euvres, III, Paris, Gallimard, coll.” Folio", 2000, 300.

Chion, Michel, Le Promeneur écoutant, essais d'acoulogie, Paris, Éditions Plume, 1993.

Chron, Michel, dans La Voix au cinéma Éditions de l’Étoile / Cahiers du cinéma, 1982, 46.

${ }^{16} C f$. notamment Peter Szendy, Écoute, une histoire de nos oreilles, précédé de Ascoltando par Jean-Luc Nancy; Jean-Luc Nancy, À l'écoute, ; Jonathan Sterne, The Audible Past. C'est Don Ihde qui, le premier, a réclamé un "tournant auditif" (auditory turn) au sein de la tradition phénoménologique (Listening and Voice. Phenomenologies of Sound), Mais l'expression semble refaire surface depuis environ une décennie - trois indices parmi tant d'autres : Nick Yablon, "Echoes of the City : Spacing Sound, Sounding Space, 1888-1916", American Literary History (où l'on peut lire : recent literary, cultural, and historical studies take what might be called an "aural turn") ; Acoustic Turn, Petra Maria Meyer (ed.), Wilhelm Fink Verlag, 2008 ; Adrienne Janus, "Listening : Jean-Luc Nancy and the "Anti-Ocular" Turn in Continental Philosophy and Critical Theory", Comparative Literature. 
DerRiDA, Jacques, "Freud et la scène de l'écriture", dans L'écriture et la différence, Seuil, 1967, 299.

DERRIDA, Jacques, Résistances - de la psychanalyse, Paris, Galilée, 1996, 102.

DerRidA, Jacques, Résistances de la psychanalyse, Paris, Galilée, 1996, 102.

HeIDEgGer, Martin, Einführung in die Metaphysik (Gesamtausgabe, II, 40), Vittorio Klostermann, 1983, 125.

HeIDEGGER, Martin, Introduction à la métaphysique, traduit de l'allemand par Gilbert Kahn, Paris, Gallimard, 1967, 125.

Нітснсоск, Alfred, Hitchcock on Hitchcock. Selected Writings and Interviews, edited by Sidney Gottlieb, University of California Press, 1997, 125 sq.

IndE, Don, Listening and Voice. Phenomenologies of Sound, State University of New York Press, 2007.

JanUS, Adrienne, "Listening : Jean-Luc Nancy and the "Anti-Ocular" Turn in Continental Philosophy and Critical Theory", Comparative Literature, vol. 63, n 2, Duke University Press, 2011.

Laderman, David, Driving Visions. Exploring the Road Movie, Austin, University of Texas Press, 2002.

Lynch, David, Entretiens avec Chris Rodley, traduit de l'anglais par Serge Grünberg, Cahiers du cinéma, 2004, 204.

Nancy, Jean-Luc, "Cinéfile et cinémonde", Trafic, n 50, p. o. 1., mai 2004.

NANCY, Jean-Luc, "Entretien sur la pluralité des mondes", dans Les Muses, Galilée, Paris, 1994, 36.

Nancy, Jean-Luc, À l'écoute, Paris, Galilée, 2002.

NANCY, Jean-Luc, L'Évidence du film. Abbas Kiarostami, Yves Gevaert éditeur, 2001, 15.

NAncy, Jean-Luc, La Déclosion (Déconstruction du christianisme, 1), Paris, Galilée, 2005, 161.

Orgeron, Devin, Road Movies: From Muybridge and Méliès to Lynch and Kiarostami, New York, Palgrave Macmillan, 2008.

Sterne, Jonathan, The Audible Past, Duke University Press, 2003.

Szendy, Peter, Écoute, une histoire de nos oreilles, précédé de Ascoltando par Jean-Luc Nancy, Paris, Minuit, 2001.

Szendy, Peter, Sur écoute. Esthétique de l'espionnage, Paris, Minuit, 2007.

YABlon, Nick, "Echoes of the City : Spacing Sound, Sounding Space, 18881916", in American Literary History, vol. 19, n 3, 2007. 